\title{
Parâmetros biofísicos na detecção de mudanças na cobertura e uso do solo em bacias hidrográficas
}

\author{
Helio L. Lopes ${ }^{1}$, Ana L. B. Candeias ${ }^{2}$, Luciano J. 0. Accioly 3 , Maria do C. M. Sobral ${ }^{4} \&$ Admilson P. Pacheco $^{2}$
}

\section{RESUMO}

0 estudo de parâmetros biofísicos como o Índice de Vegetação da Diferença N ormalizada (NDVI), albedo e temperatura da superfície (LST), aplicado a ecossistemas, tem sido relevante para 0 entendimento de mudanças relacionadas à degradação do meio ambiente. Algumas al terações que provocam desequilíbrio de interações ecológicas em ecossistemas, como o desmatamento, a mineração, a agricultura inadequada e o superpastejo, entre outros, estão interrelacionadas. Para avaliação de mudanças temporais relacionadas à degradação do ecossistema caatinga obtiveram-se as imagens da diferença de três parâmetros: NDVI, albedo e temperatura da superfície, para os anos de 1985 e 2001 (estação seca), utilizando-se imagens TM. Este estudo foi aplicado à bacia do rio Brígida onde há uma exploração intensa dos recursos naturais, em que os resultados mostram aumento na temperatura da superfície, diminuição do NDVI e pouca variação no albedo da superfície evidenciando, assim, que entre os anos de 1985 e 2001 houve avanço na degradação dos recursos naturais, nesta bacia.

Palavras-chave: temperatura da superfície, al bedo, índice de vagetação, degradação da caatinga, modelagem de dados

\section{Biophysical parameters in the detection of changes in soil cover and use in watersheds}

\begin{abstract}
The study of biophysical parameters such as NDVI, albedo and surface temperature has been reported as important for the understanding of land degradation changes of ecosystems. Degradation in ecosystems is related to the inadequate use of the environmental resources including deforestation, mining, inadequate agriculture, overgrazing, among others, that cause imbalance of ecological interactions. For multitemporal evaluation of changes related to the degradation of the 'caatinga' ecosystem three parameters were used with TM images: NDVI, albedo and surface temperature, in two different dates, 1985 and 2001 (dry season). This study was applied in the Brígida river basin where there is a great intensification in the exploration of the natural resources. The results show an increase in the surface temperature, decrease of NDVI and little variation in the surface albedo, between the two dates. These results also show an increase in the degradation of the natural resources in the basin of the Brígida river.
\end{abstract}

Key words: surface temperature, surface albedo, vegetation index, degradation of ecosystems, data modelling

\footnotetext{
${ }^{1}$ Engenharia Agrícola e Ambiental/U NIVASF. CEP 56304-205, Petrolina, PE. Fone: (87) 3986-3802. Email: helio.lopes@univasf.edu.br ${ }^{2}$ Departamento de Engenharia Cartográfica/UFPE. CEP 51000-000, Recife, PE.. Fone: (81) 2126-8983. Email: analucia@ufpe.br; admilpp@ @ufpe.br 3 Embrapa Solos/UEP CEP 51020-240. Recife, PE. Fone: (81) 3325-5988. Email: oaccioly@uep.cnps.embrapa.br

${ }^{4}$ Departamento de Engenharia Civil/UFPE. Fone: (81)2126-8219. Email: msobral@ufpe.br
} 


\section{INTRODUÇÃO}

No seu capítulo 12, a agenda 21 define desertificação como a degradação da terra nas regiões áridas, semiáridas e subúmidas secas, resultante de vários fatores, entre eles as variações climáticas e as atividades humanas (UN, 2001). Entende-se a degradação da terra como a degradação dos solos e recursos hídricos, da vegetação e biodiversidade e a redução da qualidade de vida das populações afetadas (Araújo et al., 2002).

A desertificação afeta um sexto da população mundial, $70 \%$ das terras áridas e um terço do total de terras do planeta. O maior impacto referente à desertificação, além de espalhar a pobreza, é a perda anual de 3,5 a 4,0 milhões de hectares de áreas agricultáveis, em função de vários processos de degradação do solo no mundo (UN, 1998).

Apesar da seriedade dos impactos ambientais e socioeconômicos da desertificação, poucos esforços têm sido desenvolvidos para gerar técnicas de monitoramento e de diagnóstico na avaliação da situação e da tendência a desertificação (Rubio \& Bochet, 1998).

Durante décadas, o papel de técnicas espaciais no monitoramento e no mapeamento da desertificação vem sendo examinado por muitos pesquisadores através de diversos parâmetros (García et al., 2008; Hein \& Ridder, 2006; Hountondji et al., 2006; Leibovici et al., 2007; Rahman et al., 2007). Imagens multitemporais de satélites em conjunto com dados locais e mapas têm sido usados para examinar a natureza, tendência e quantificação do processo de desertificação em diferentes ambientes (Carvalho, 2001), incluindo áreas do bioma caatinga (Lopes et al., 2009; Souza et al., 2004). O delineamento e mapeamento de áreas afetadas são realizados visando estabelecer uma base confiável para o monitoramento do processo de desertificação e estratégias adequadas para combatê-la.

A desertificação gera problemas sociais, econômicos e ambientais, visto que a recuperação de áreas degradadas é demorada e dispendiosa. Diante desta realidade o programa da ONU ressalta, para combate à desertificação, a realização de pesquisas envolvendo técnicas espaciais utilizando parâmetros biofísicos, para diagnosticar e monitorar áreas sob risco de desertificação e em processo de desertificação (UN, 2001).

Os estudos sobre indicadores do processo de desertificação são necessários na detecção de mudanças e é imprescindível que estejam entre as prioridades da pesquisa, devendo subsidiar a tomada de decisões na esfera político-administrativa, apresentar condições para se estabelecer prioridades de ações quanto às medidas de prevenção, reabilitação e recuperação de terras degradadas e definir novas linhas de pesquisa (Araújo et al., 2002).

Indicadores são integrados a informações sintéticas que podem prover dados em estágio inicial, situação e evolução de processos físicos, químicos, biológicos e antropogênicos na dinâmica de ecossistemas. Yan et al. (2007) utilizaram imagens TM Landsat de diferentes datas para verificar mudanças no uso e cobertura do solo, classificando o estágio da degradação em quatro níveis, classificação, também, utilizada por Candido et al. (2002), que a classificaram em quatro níveis de degradação ambiental: baixo, moderado, grave e muito grave utilizando, além de imagem, dados de solo, de topografia, sociais e manejo do solo.

A quantificação, a avaliação de risco e o monitoramento da desertificação, podem ser realizados a partir de parâmetros biofísicos (tais como, índices de vegetação) e físicos (albedo, temperatura, emissividade, dentre outros) obtidos por meio de imagens orbitais, para determinação de mudanças na superfície. Julien \& Sobrino (2009) utilizaram o NDVI e a LST para determinarem parâmetros da superfície, como a umidade do solo, e mudanças na superfície. Para Liu et al. (2006), a temperatura da superfície pode ser um parâmetro chave para diversos estudos do meio ambiente. Herb et al. (2008) desenvolveram um modelo para determinação da temperatura da superfície visando a identificação dos diversos usos e cobertura da superfície, o qual pode ser usado para diferenciar mudanças na superfície. A avaliação de dados históricos da precipitação, segundo Souza et al. (2004), e da evapotranspiração também pode auxiliar nesta identificação e em sua quantificação (Basstiaansen, 1998; Souza et al., 2004; UN, 2001).

Series do NDVI e LST também podem ser utilizadas para determinar mudanças na vegetação (Julien et al., 2006). Os resultados apresentados por esses autores, mostram diminuição do NDVI em algumas regiões e aumento em determinados locais, além de um aumento na temperatura da superfície maior que $2,5^{\circ} \mathrm{C}$, evidenciando que as áreas áridas e semiáridas se tornaram mais áridas.

Objetivo com este trabalho avaliar a importância dos parâmetros de temperatura da superfície, albedo e NDVI (Normalized Difference Vegetation Index) na quantificação das mudanças ocorridas entre os anos de 1985 e 2001, na área afetada pelos processos de desertificação na Bacia do Rio Brígida (Pernambuco), por meio de imagens do sensor TM - Landsat 5.

\section{MATERIAL E MÉTODOS}

\section{Área de estudo}

A Bacia do Rio Brígida foi escolhida devido à intensidade de exploração do ecossistema da região pela indústria do gesso, pela agricultura de sequeiro, pelas olarias, pelas pastagens e pelo fato de que alguns municípios ultrapassam o limite de densidade demográfica estabelecido pela ONU para regiões susceptíveis a desertificação, que é de $20 \mathrm{hab} \mathrm{km}^{-2}$ havendo, assim, uma sobrecarga no ecossistema caatinga.

A área selecionada está localizada no oeste do Estado de Pernambuco, na parte central do Trópico semiárido brasileiro. A Bacia do Rio Brígida faz parte da mesorregião do Sertão Pernambucano, área em que estão situados 6 municípios com área total incluída na bacia (Tabela 1). 
Tabela 1. Alguns dados dos municípios da Bacia do Rio Brígida e coordenadas geográficas das suas sedes (datum WGS-84)

\begin{tabular}{cccc}
\hline Municípios & Área $\left.\mathbf{( k m}^{2}\right)$ & População & Altitude $(\mathbf{m})$ \\
Araripina & 1914,40 & 70.592 & 622 \\
Bodocó & $1.604,90$ & 31.712 & 443 \\
Granito & 519,70 & 6.104 & 447 \\
Ipubi & 972,10 & 23.210 & 535 \\
Ouricuri & 2383,90 & 56.623 & 451 \\
Trindade & 229,30 & 21.919 & 518 \\
\hline
\end{tabular}

Outros municípios e parte de seus territórios inseridos na Bacia, são: Cabrobó, Exu, Moreilândia, Orocó, Parnamirim, Santa Maria da Boa Vista, Santa Cruz, Santa Filomena e Serrita.

A Bacia está localizada entre as coordenadas $7^{\circ} 18^{\prime} 57^{\prime \prime}$ e $8^{\circ} 36^{\prime} 30^{\prime \prime}$ de latitude Sul e $39^{\circ} 17^{\prime} 16^{\prime \prime}$ e $40^{\circ} 43^{\prime} 30^{\prime \prime}$ de longitude Oeste e ocupa uma área de aproximadamente $14000 \mathrm{~km}^{2}$ (Figura 1).

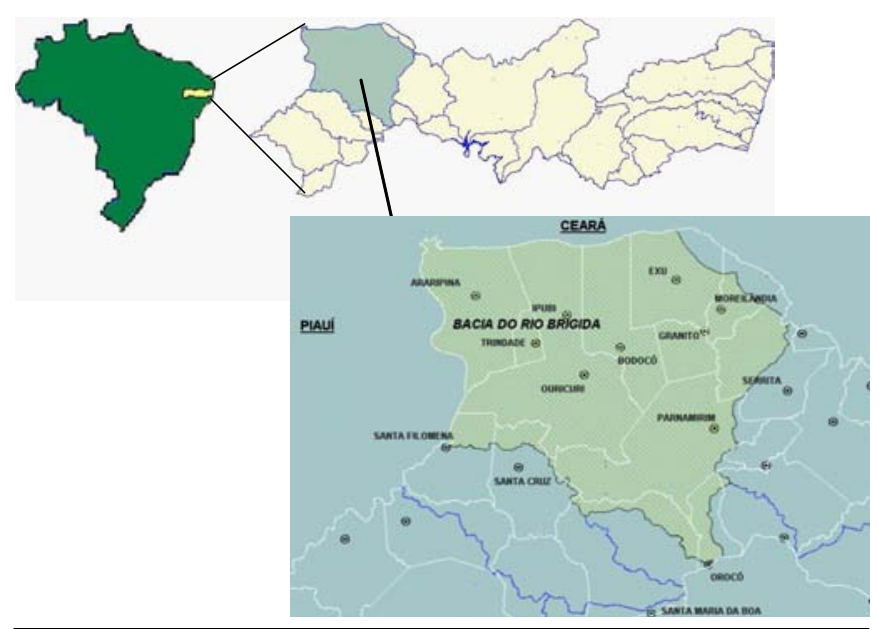

Figura 1. Localização da área de estudo

\section{Cálculo do NDVI, albedo e temperatura da superfície}

As imagens TM foram georreferenciadas pelo sistema de coordenadas UTM e datum SAD69 e realizado o mosaico das seguintes cenas: 217-65 e 217-66 de 24 de outubro de 1985 e 04 de outubro de 2001, respectivamente. A modelagem dos parâmetros biofísicos foi realizada em Linguagem Espacial para Geoprocessamento Algébrico-LEGAL, disponível no SPRING 5.0.

Para determinação do NDVI calculou-se, inicialmente, a radiância (Eq. 1) e, em seguida, a reflectância (Eq. 2) para as bandas 3 e 4 do sensor TM - Landsat 5 .

$$
\mathrm{L}_{\mathrm{rad}}=\mathrm{L}_{\mathrm{i}, \text { min }}+\left(\frac{\mathrm{L}_{\mathrm{i}, \text { max }}-\mathrm{L}_{\mathrm{i}, \text { min }}}{255}\right) \times \mathrm{NC}_{\mathrm{i}}
$$

em que: $\mathrm{L}_{\mathrm{i}}$ é a radiância espectral na banda i em $\mathrm{Wm}^{-2} \mathrm{sr}^{-1} \mu \mathrm{m}^{-1}$; $\mathrm{L}_{\mathrm{i} \text {,max }}$ e $\mathrm{L}_{\mathrm{i}, \min }$ os valores de radiância máxima e mínima na banda $\mathrm{i}$, respectivamente e $\mathrm{NC}_{\mathrm{i}}$ o nível de cinza na banda $\mathrm{i}$.

$$
\mathrm{r}_{\mathrm{o}, \mathrm{i}}=\frac{\mathrm{L}_{\mathrm{rad}} \times \pi}{\mathrm{E}_{\mathrm{o}, \mathrm{i}} \times \cos \theta \times \mathrm{d}_{\mathrm{r}}}
$$

em que:

$\mathrm{r}_{\mathrm{o}, \mathrm{i}}$ é a reflectância espectral na banda i

dr o inverso do quadrado da distância Terra-Sol em unidade astronômica

$\mathrm{E}_{\mathrm{o}, \mathrm{i}} \mathrm{O}$ valor médio da irradiância solar exoatmosférica na banda i em $\mathrm{W} \mathrm{m}^{-2} \mu \mathrm{m}^{-1}$ (constante solar)

q, o ângulo zenital solar (calculado a partir da informação do ângulo de elevação solar, b, disponível no arquivo cabeçalho da imagem: $\mathrm{q}=\left(90^{\circ}-\mathrm{b}\right)$ e Li é a radiância espectral na banda i em $\mathrm{Wm}^{-2} \mathrm{sr}^{-1} \mu \mathrm{m}^{-1}$.

Em seguida, realizou-se a correção atmosférica pelo método DOS (Dark Object Subtraction), usando-se o histograma de cada banda para selecionar o pixel mais escuro (Gürtler et al., 2005). Não é incluído o albedo da superfície em que a correção atmosférica é determinada pelo albedo atmosférico e pela transmitância atmosférica (Bastiaanssen \& Bakker, 2000).

Desta forma, o NDVI é dado pela seguinte equação (Qi et al., 1994)

$$
\mathrm{NDVI}=\frac{\mathrm{r}_{\mathrm{NIR}}-\mathrm{r}_{\text {RED }}}{\mathrm{r}_{\mathrm{NIR}}+\mathrm{r}_{\mathrm{RED}}}
$$

donde $\mathrm{r}_{\mathrm{NIR}}$ é a reflectância no infravermelho e $\mathrm{r}_{\mathrm{RED}}$ é a reflectância no vermelho.

No cálculo da temperatura da superfície, conforme a Eq. 4 (Bastiaanssen \& Bakker (2000), utilizou-se a banda termal do TM calculando-se a radiância para a respectiva banda e a emissividade de acordo com a fórmula 5 .

$$
\mathrm{LST}=\frac{1260}{\ln \left(\frac{61,6}{\mathrm{~B}_{\mathrm{TM} 6}}+1\right)} / \varepsilon^{0,25}
$$

donde:

LST - Temperatura da superfície $\mathrm{K}$

$\mathrm{B}_{\text {TM6- }}$ Radiância termal $\mathrm{Wm}^{-2} \mathrm{sr}^{-1} \mu \mathrm{m}^{-1}$

$\varepsilon \quad$ - Emissividade da superfície

$$
\varepsilon_{(8-14)}=1,009+0,047 \mathrm{Ln}(\mathrm{NDVI})
$$

O albedo foi calculado pela fórmula 6 (Bastiaanssen \& Bakker, 2000) através das bandas de 1 a 5 e 7 do Thematic Mapper.

$$
r_{o}=\frac{r_{p}-r_{a}}{\tau_{s w}}
$$

em que:

$r_{p}$ - albedo no topo da atmosfera

$r_{a}$ - albedo da atmosfera

$\tau_{\mathrm{sw}}$ - transmitância atmosférica.

Para avaliação das mudanças entre os anos de 2001 e 1985 realizaram-se a diferença das imagens NDVI, Albedo e Temperatura da superfície e a análise dos histogramas dessas imagens para cada um desses anos. 


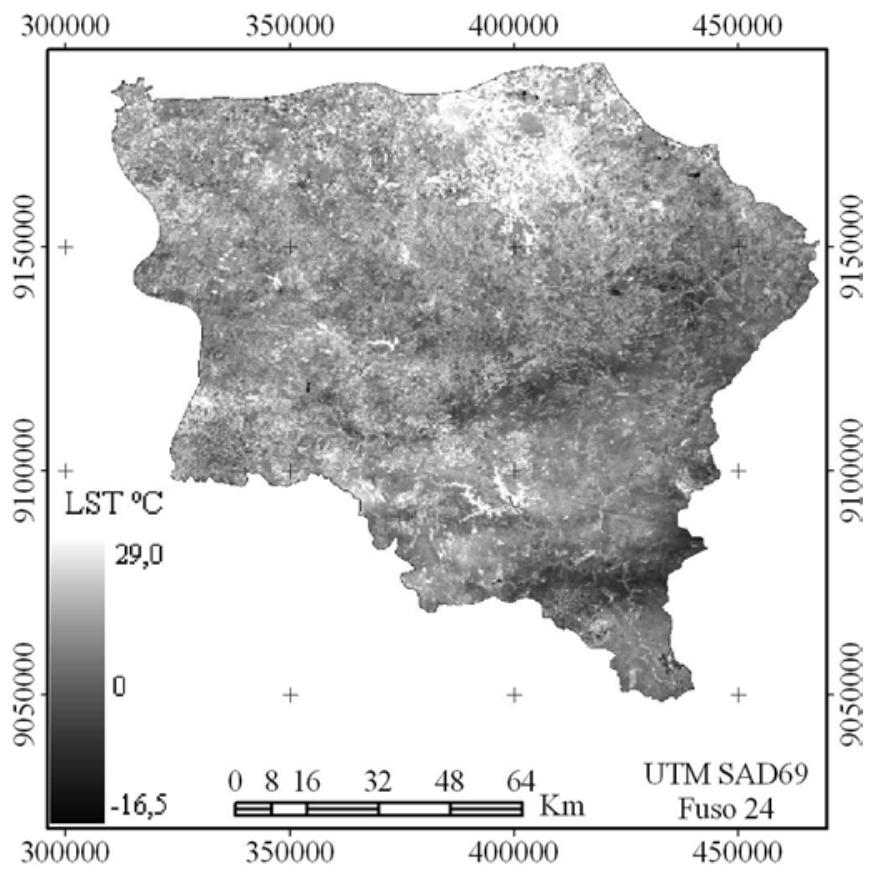

Figura 2. Imagem da diferença da temperatura da superfície dos anos de 2001 e 1985

\section{RESULTADOS E DISCUSSÃO}

A Figura 2 mostra a imagem da diferença da temperatura em que do ponto cinza (0) para o mais escuro mostra áreas com dimi- nuição da temperatura da superfície; do cinza (0) para o ponto mais claro indica áreas com aumento na temperatura da superfície.

Na Figura 2 se percebe aumento da temperatura da superfície em torno de $12{ }^{\circ} \mathrm{C}$ em média, onde antes havia uma vegetação arbórea densa (região norte) que foi substituída por solo exposto devido ao intenso desmatamento; aumento semelhante foi encontrado para o leito seco dos açudes que, em 1985, estavam com um nível de espelho d'água considerável. $\mathrm{O}$ valor $-16,5^{\circ} \mathrm{C}$ na imagem corresponde à nuvem na imagem de 2001, e solo exposto em 1985 e o valor de $29,0{ }^{\circ} \mathrm{C}$ na imagem corresponde ao solo exposto com ocorrência de queimada em 2001 (Figura 3), e vegetação densa na imagem de 1985 (Figura 3).

Valores por volta de $-4,5{ }^{\circ} \mathrm{C}$ ocorreram em locais com surgimento de novos reservatórios de água (açudes e barreiros) (Figura 4), e na região sul, onde as chuvas ocorridas nos municípios de Ouricuri e Cabrobó no período da passagem do satélite, responderam por esta redução. Observa-se, também, nas imagens, em que existia vegetação na imagem de 1985 e agora solo exposto na imagem de 2001 , aumento em torno de $11{ }^{\circ} \mathrm{C}$; aumento de $7{ }^{\circ} \mathrm{C}$ (canto superior direito da Figura 4), embora permanecesse com vegetação, ocorreu devido à maior fragmentação do remanescente da caatinga; com isto, aumenta o efeito de borda, prevalecendo espécies pioneiras, mudando o microclima no fragmento (Denyer et al., 2006). A precipitação nesses municípios nos quatro meses que antecederam a aquisição das imagens de 1985, ficou em torno
A.

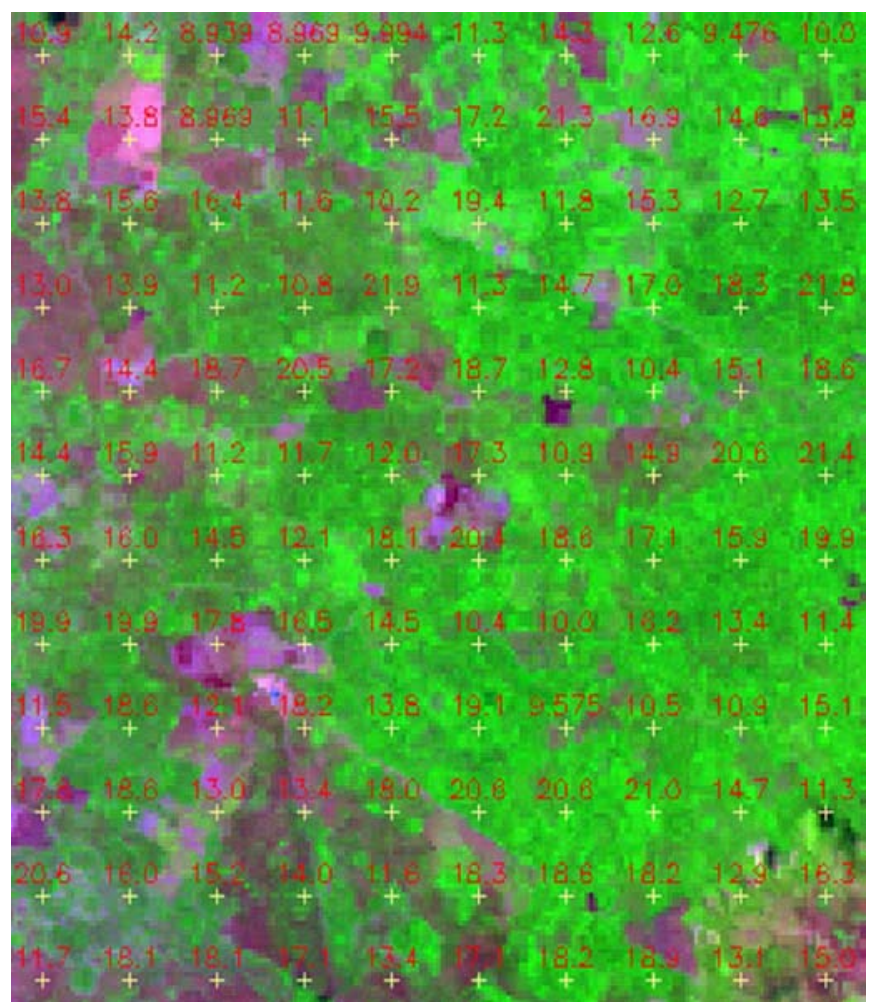

B.

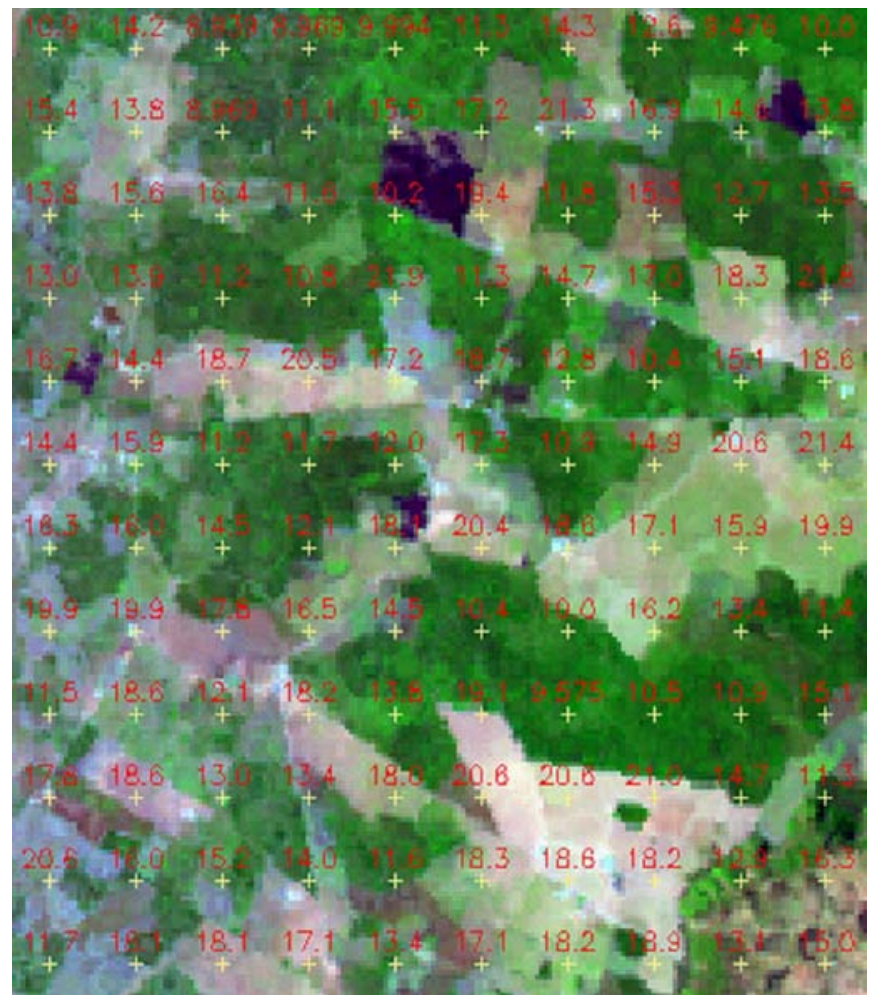

Figura 3. Variação espaço-temporal da temperatura da superfície na porção norte da bacia. A) Imagem Landsat TM 5 composição RG B 543 de outubro de 1985 , com sobreposição da grade da diferença da temperatura da superfície, e B) Imagem Landsat TM 5 composição RGB 543 de outubro de 2001, com sobreposição da grade da diferença da temperatura da superfície 
A.

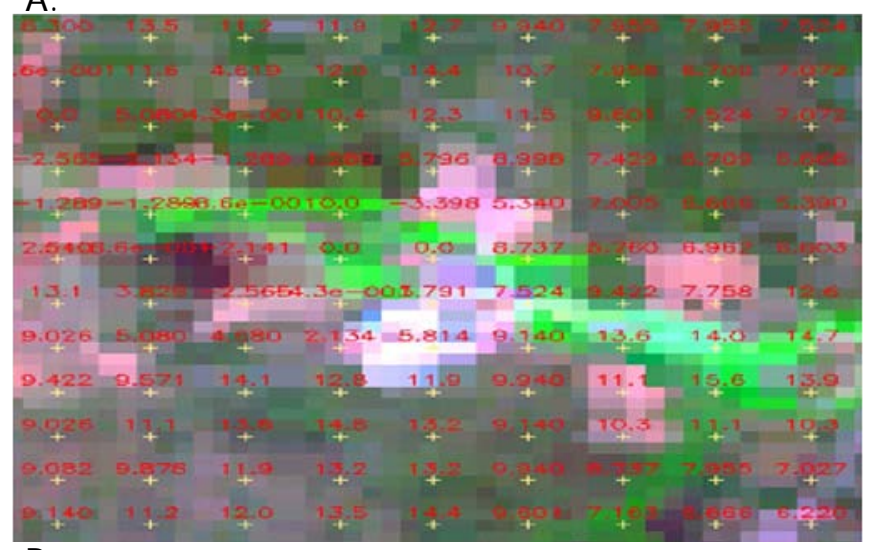

B.

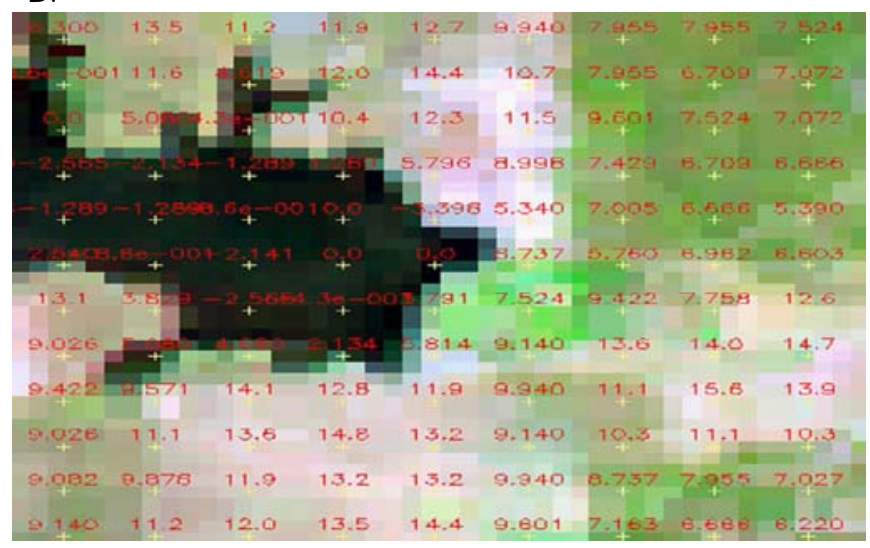

Figura 4. Variação espaço-temporal da temperatura da superfície na porção sul da bacia. A) Imagem Landsat TM 5 composição RGB 543 de out. de 1985, com sobreposição da grade da diferença da temperatura da superfície; B) Imagem LandsatTM 5 composição RGB 543 de out. de 2001, com sobreposição da grade da diferença da temperatura da superfície

Tabela 2. Valores da precipitação para os quatro meses antes da tomada da imagem e a soma para o mesmo período para os municípios de Cabrobó e O uricuri, Pernambuco. Dados: DISME/ Recife

\begin{tabular}{|c|c|c|c|c|c|}
\hline \multirow{2}{*}{ Mês } & Cabrobó & Ouricuri & \multirow{2}{*}{ Mês } & Cabrobó & Ouricuri \\
\hline & \multicolumn{2}{|c|}{1985} & & \multicolumn{2}{|c|}{2001} \\
\hline 07 & 20,50 & 24,50 & 06 & - & 2,10 \\
\hline 08 & 7,80 & 6,60 & 07 & 20,40 & 21,90 \\
\hline 09 & 0,70 & 3,40 & 08 & 5,30 & 3,90 \\
\hline 10 & 13,00 & 4,40 & 09 & 0,00 & 0,00 \\
\hline Total & 42,00 & 38,90 & Total & 25,70 & 27,90 \\
\hline
\end{tabular}

de $40 \mathrm{~mm}$ e $30 \mathrm{~mm}$ para 2001, distribuídos conforme Tabela 2 porém as precipitações não são uniformes na região, podendo ocorrer em pontos localizados.

Um acúmulo de $30 \mathrm{~mm}$ é suficiente para o surgimento do ciclo fenológico com o aparecimento de folhas em algumas espécies de arbustos e de plantas lenhosas e herbáceas. A cobertura verde ocorre aproximadamente $20 \mathrm{a}$ 60 dias após o início da estação chuvosa período em que, dependendo da espécie, esta cobertura pode ser superior a 90\% (Barbosa et al., 1989; Pereira et al., 1989). Verificou-se que na região os dados de precipitação são escassos, necessitando-se de um desenvolvimento de metodologias (modelos) que supere esta falta de dados, associando uso do solo, pedologia e resposta espectral dos alvos.

Os valores acima de $20^{\circ} \mathrm{C}$ estão relacionados com exposição do solo e com afloramento rochoso. Nos histogramas percebe-se melhor a variação da temperatura para as duas épocas (Figura 5).

Para 1985, o referido histograma revelou que a maior parte dos pixels tinha temperaturas em torno de 16 e 19 ${ }^{\circ} \mathrm{C}$ (Figura 5). No ano de 2001, que também foi analisado na estação seca, a maioria dos pixels apresentou temperaturas por volta de $29^{\circ} \mathrm{C}$, resultados que indicam aumento por volta de $10{ }^{\circ} \mathrm{C}$ na temperatura da superfície na região norte da bacia do rio Brígida, no período considerado. Atribuiu-se este aumento às alterações na cobertura provocadas pela expansão das atividades do polo gesseiro do Araripe, à intensificação de agricultura de sequeiro e ao desmatamento com práticas de queimadas. Temperaturas acima de $40{ }^{\circ} \mathrm{C}$ correspondem à ocorrência de queimadas, prática comum na agricultura de sequeiro que ocorre na região.

Com a intensificação no uso dos recursos naturais na Bacia em estudo como, por exemplo, recursos florestais para a produção de carvão, com o total de $569.079 \mathrm{~m}^{3}$ estimados, $156.708 \mathrm{~m}^{3}$ amostrados e $125.468 \mathrm{~m}^{3}$ com origem identificada (Silva et al., 1998), para a microrregião de Araripina e indústria do gesso, de 1985 a 2000, deu-se um aumento no beneficiamento de $1113,72 \%$, conforme esquematizado no gráfico da Figura 6. Pela análise dos parâmetros foi grande a degradação do ecossitema da bacia, não refletindo no aumento proporcional do beneficiamento da gipsita em função da gpsita bruta, o que pode ocasionar o não divulgamento do valor real da gipsita processada, pois acarreta consumo de produtos florestais. O mapeamento dessas áreas é importante para uma análise local do nível de degradação da referida região, servindo de indicação para o direcionamento de políticas públicas de gestão ambiental e na avaliação do consumo de produtos florestais.

Os valores de temperatura máxima e mínima, o desvio padrão e a média para toda a bacia, foram calculados para se comparar os dados estatísticos das duas épocas (Tabela 3), referentes a uma área sem influência de rocha, sombra de nuvem, nuvens e corpos d'água, conforme Figura 5.

Conforme a Tabela 3, no ano de 1985 a temperatura da superfície mínima foi de $16,18{ }^{\circ} \mathrm{C}$, para uma região localizada na chapada do Araripe, no município de Exu, onde ocorre vegetação do tipo arbórea densa, que indica área preservada (alvo pseudo-invariante). $\mathrm{O}$ valor da temperatura máxima foi de $28,46{ }^{\circ} \mathrm{C}$, para uma área com ocorrência de solo exposto. $\mathrm{O}$ desvio padrão da temperatura para o ano de 1985 foi de $2,03{ }^{\circ} \mathrm{C}$ e a média da temperatura foi de $19,34{ }^{\circ} \mathrm{C}$. Na imagem de 2001, para uma área localizada na região norte a temperatura mínima foi de $26,78{ }^{\circ} \mathrm{C}$. A maior temperatura foi de $45,16{ }^{\circ} \mathrm{C}$ e esta área está localizada numa propriedade com ocorrência de solo exposto e queimada. $\mathrm{O}$ desvio padrão da temperatura para este ano foi de $3,77^{\circ} \mathrm{C}$ e a temperatura média, de $32,04{ }^{\circ} \mathrm{C}$. 

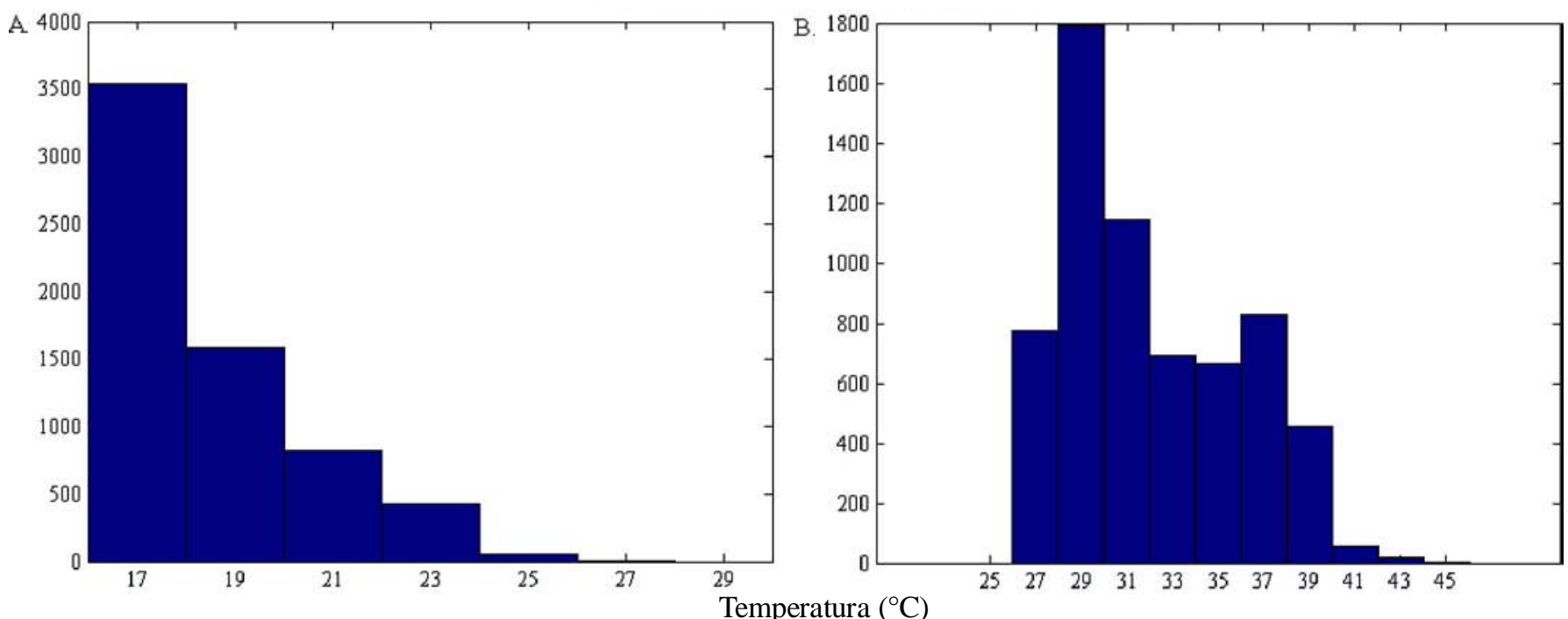

Figura 5. Distribuição para um intervalo de 2 em 2 C. da temperatura da superfície na região norte bacia do rio Brígida. A) ano de 1985 e B) ano de 2001

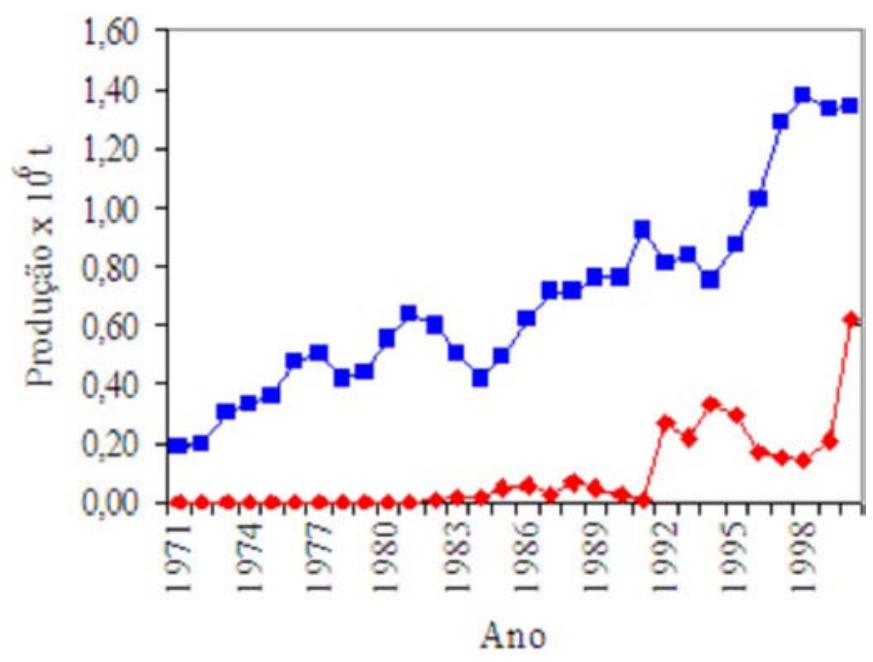

$\rightarrow$ Gipsita bruta $\rightarrow$ Gipsita beneficiada

Figura 6. Evolução da exploração e beneficiamento da gipsita na microrregião de Araripina. FONTE: Santos, 2006

Tabela 3. Valores de mínimo e máximo, desvio padrão e média para a temperatura da superfície

\begin{tabular}{lcc}
\hline \multirow{2}{*}{ Ano } & Estatísticas para a Temperatura da Superfície (ㅇ) \\
\cline { 2 - 3 } Mín & $\mathbf{1 9 8 5}$ & $\mathbf{2 0 0 1}$ \\
Máx & 16,18 & 26,78 \\
Desvio Padrão & 28,46 & 45,16 \\
Média & 2,03 & 3,77 \\
\hline
\end{tabular}

Para a imagem da diferença do NDVI, as áreas do cinza (0) para o branco representam aumento no NDVI e as que têm amplitude do cinza (0) para o mais escuro, são regiões nas quais ocorreu diminuição no NDVI (Figura 7).

Constatou-se, na Figura 7, aumento em torno de 0,27 no NDVI na região sul da bacia, nos municípios de Cabrobó e Orocó, região que pertence ao núcleo de deserti-

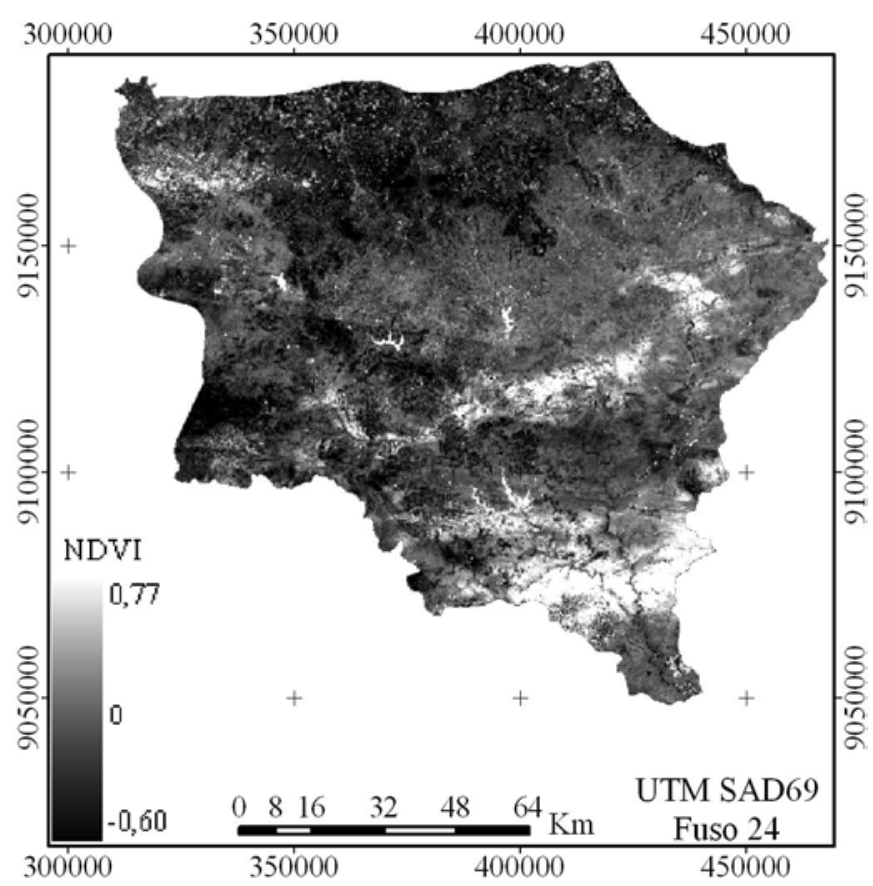

Figura 7. Imagem da diferença do NDVI para os anos de 2001 e 1985

ficação de Cabrobó e onde houve precipitação em torno de $25 \mathrm{~mm}$, conforme Tabela 2, havendo influência no resultado já que espécies colonizadoras que representam uma sucessão secundária podem rebrotar de imediato, à menor ocorrência de chuvas, tendo como exemplos a jurema preta e o marmeleiro (Pereira et al., 1989; Barbosa et al., 1989). Valores por volta de $-0,30$ ocorreram na região oeste da bacia na divisa dos municípios de Araripina e Ouricuri; nesta região há presença de agricultura e exploração do gesso, contribuindo para o alto valor encontrado na imagem da diferença do NDVI.

Tem-se, a seguir, a distribuição do NDVI em histogramas para os anos de 2001 e 1985 , mostrando a variação em pixel para uma região a sudoeste da bacia (Figura 8). 

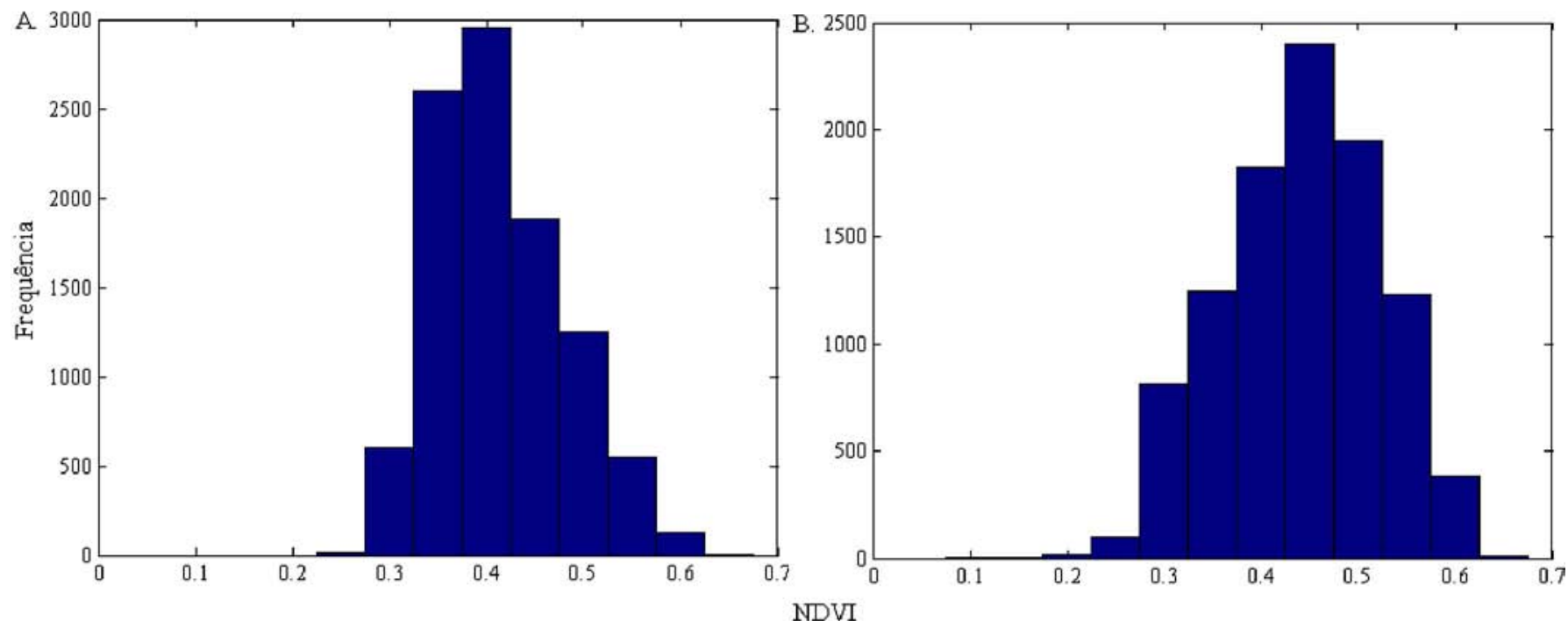

Figura 8. Distribuição dos valores do NDVI para parte da região sudoeste da bacia com referência às duas imagens analisadas. A) ano de 1985 e B) ano de 2001

A.

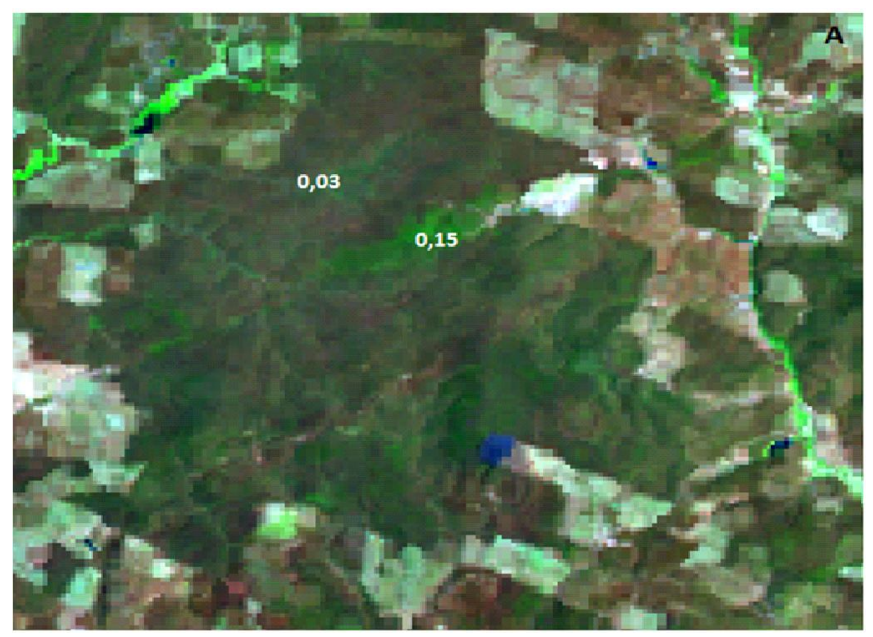

B.

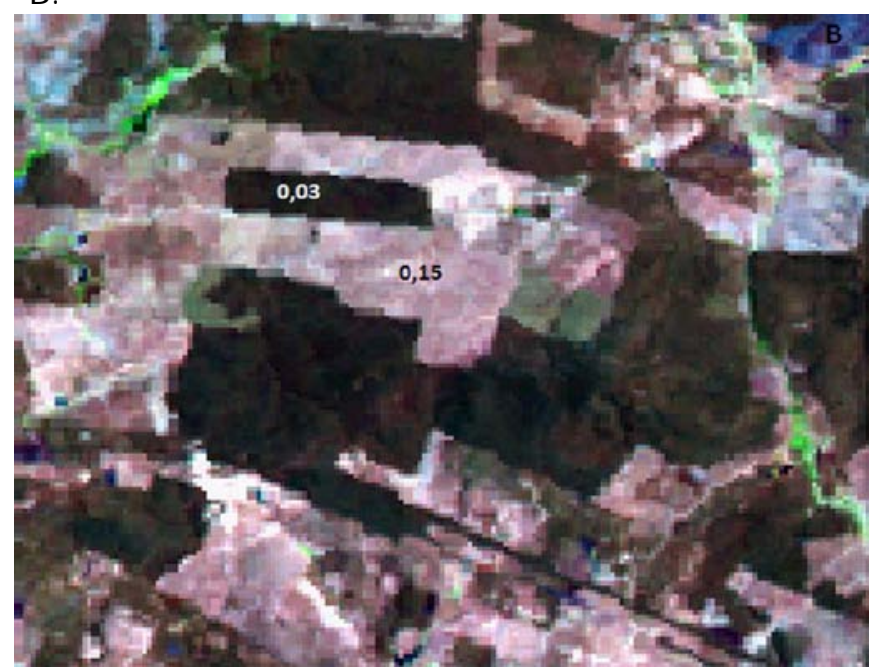

Figura 9. Composição RGB 543 de imagens Landsat TM 5 com sobreposição da grade da diferença do NDVI. A) referente ao ano de 1985 e B) referente ao ano de 2001
Conforme os histogramas da Figura 8, a maior parte apresentou valores de NDVI entre 0,35 a 0,40 totalizando 5500 pixels; para 2001, uma grande quantidade de pixel indicou valores de NDVI entre 0,40 e 0,50, ficando por volta de 6000 pixels perfazendo, assim, aumento no valor do NDVI, apesar de ter ocorrido mudança no uso do solo com supressão da caatinga e ocorrência de solo exposto, conforme visto no recorte da imagem apresentada na Figura 8 , que pode ter acontecido em virtude das precipitações na região e predominância de dossel herbáceo; observa-se, também, o surgimento de pixels com valores abaixo de 0,15 , referentes a desmatamento, que resultou em diminuição do NDVI, conforme visto na Figura 9.

Observam-se, na Figura 9, mudanças da cobertura e uso do solo, refletindo na diminuição do NDVI por volta de 0,15 , e nos locais que permaneceram com vegetação ocorreu diminuição do NDVI, que variou de 0,01 a 0,03 ; essa variação é mínima e pode estar relacionada com a fragmentação do ecossistema, como visto nas imagens.

Realizaram-se análises dos valores de mínimo e máximo, desvio padrão e média relativos ao NDVI para as duas imagens estudadas, referente a uma região no sudeste da bacia (Tabela 4).

De acordo com a Tabela 4, o valor mínimo de NDVI para o ano de 1985 foi 0,25 ; a área com este valor está localizada no município de Orocó, com uso agrícola; o maior valor de NDVI ocorreu próximo a um riacho, no município de Parnamirim, onde existe uma vegetação arbustiva arbórea aberta; o desvio padrão para a imagem do NDVI de 1985 foi de 0,06 e a média, de 0,41 . Na análise dos valores para a imagem de 2001 a área com menor valor está localizada em locais com solo exposto; o maior valor para esta imagem foi de 0,65 e o pixel com este valor fica em áreas agrícolas, no município de Orocó. O desvio padrão para esta imagem foi de 0,08 e a média, de 0,44 . Os valores negativos do NDVI referiram a nuvens e água. Determinou-se, para essas áreas uma máscara; os 

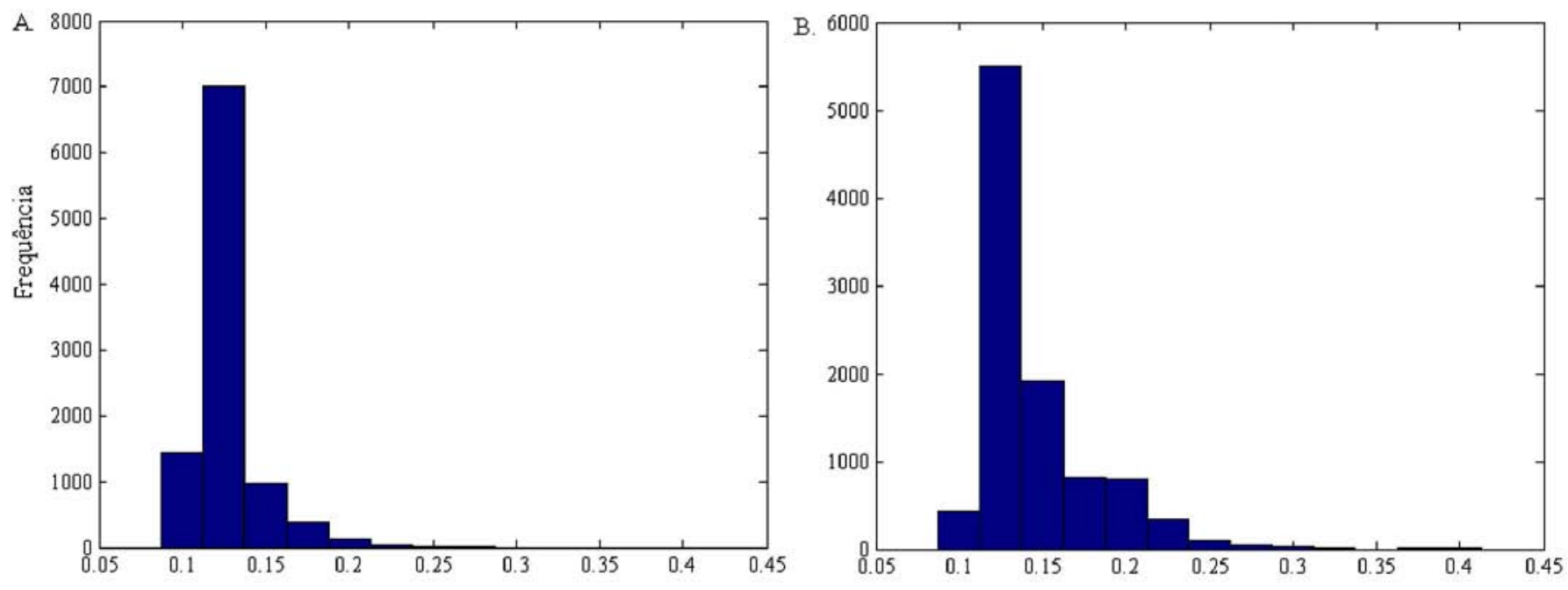

Albedo

Figura 10. Distribuição dos valores do al bedo para parte da região sudeste em relação as imagens analisadas. A) para 0 ano de 1985 e B) para 0 ano de 2001

Tabela 4. Valores de mínimo e máximo, desvio padrão e média para 0 NDVI

\begin{tabular}{lll}
\hline \multirow{2}{*}{ Ano } & \multicolumn{2}{c}{ Estatísticas para o NVDI } \\
\cline { 2 - 3 } Mín & $\mathbf{1 9 8 5}$ & $\mathbf{2 0 0 1}$ \\
Máx & 0,25 & 0,11 \\
Desvio Padrão & 0,63 & 0,65 \\
Média & 0,06 & 0,08 \\
\hline
\end{tabular}

outros valores negativos foram aproximados para zero com vista à aplicação da fórmula da emissividade.

Com relação ao albedo apresenta-se a seguir (Figura 10) a distribuição dos valores do albedo calculados para parte da região sudeste da bacia para as datas avaliadas.

Tabela 5. Valores de mínimo e máximo, desvio padrão e média do albedo, referentes a uma área na região sudeste da bacia

\begin{tabular}{lll}
\hline \multirow{2}{*}{ Ano } & \multicolumn{2}{c}{ Estatísticas para o NVDI } \\
\cline { 2 - 3 } Mín & $\mathbf{1 9 8 5}$ & $\mathbf{2 0 0 1}$ \\
Máx & 0,09 & 0.09 \\
Desvio Padrão & 0,24 & 0,42 \\
Média & 0,02 & 0,03 \\
\hline
\end{tabular}

Verifica-se que, em 1985, a maioria dos pixels indicou valores de albedo em torno de 0,12 , representando região com caatinga densa; valores em torno de 0,30 se referem a solos arenosos expostos. Para o histograma de 2001, é observado um deslocamento dos pixels de 0,12 para o valor de 0,15 , ocorrendo pouca diferença na variação do albedo; para o albedo foi pouca a variação nos dados fato que pode ser devido à influência da precipitação (Tabela 2), indicando que, para o albedo, a precipitação exerce uma influência maior, tornando-a mais homogênea.
A Tabela 5, a seguir, mostra os valores de mínimo e máximo, desvio padrão e média, para o albedo nos períodos analisados.

Um valor de 0,24 foi encontrado na região de solo exposto para a imagem albedo de 1985 , representando o valor máximo; para o ano de 1985, o albedo mostrou desvio de 0,02 e média de 0,12 ; emm 2001, os valores mais altos do albedo ficaram em torno de 0,42 , localizados entre os municípios de Orocó e Cabrobó; neste ano, a imagem albedo teve desvio padrão de 0,03 e média de 0,14; para esta região, onde houve albedo em torno de 0,42 , há solo exposto devido à exploração agrícola e de recursos florestais. Os valores mínimos apresentados na Tabela 5 são para água, que serviu de correção atmosférica, realizada na equação do albedo (Bastiaanssen \& Bakker, 2000), e valores de 0,11 representam vegetação arbórea densa localizada nos topos de serras.

\section{CONCLUSÕES}

1. Através dos parâmetros biofísicos analisados neste estudo, verifica-se o forte impacto da antropização na região.

2. A metodologia mostrou-se eficiente na realização dos estudos da degradação ambiental, além de evolução dos parâmetros biofísicos analisados relacionados com o uso e cobertura do solo.

3. As regiões com menor aumento na temperatura da superfície se compunham de áreas com vegetação densa do tipo vegetação arbustiva arbórea fechada e vegetação de contato entre caatinga e cerrado, que ocorrem na chapada do Araripe.

4. As regiões com grande aumento na temperatura da superfície têm, como uso em 2001, solo exposto, que correspondem a neossolos litólicos com presença de matacoes, significando um desmatamento e início de degradação do solo. Na imagem de 1985 essas áreas aparecem 
com caatinga densa e estão localizadas nos municípios de Exu e Granito.

5. Verifica-se maior influência da precipitação no NDVI que na temperatura da superfície.

6. Para o monitoramento da dinâmica do uso do solo em bacias hidrográficas através de sensoriamento remoto orbital, deve-se utilizar dados com o mínimo de influência da precipitação.

7. Comparando-se os índices estudados em escala de influência da precipitação, primeiro vem o albedo (reflectância), NDVI (reflectância), e temperatura da superfície (emitância), necessitando-se de estudos sobre o comportamento dos parâmetros em função dos níveis de precipitação.

\section{LITERATURA CITADA}

Araújo, A.; Santos, M. F. A.; Meunier, I.; Rodal, M. J. Desertificação e seca. Contribuição da ciência e tecnologia para a sustentabilidade do Semi-árido do Nordeste do Brasil, Recife: 2002. 63p.

Barbosa, D. C. de A.; Alves, J. L. H.; Prazeres, S. de M.; Paiva, A. M. A. Dados fenológicos de 10 espécies arbóreas de uma área de caatinga (Alagoinha-PE). Acta Botânica Brasileira, v.3, n.2, p.109-117, 1989.

Bastiaanssen, W. Remote sensing in water resources management: The state of the art. Colombo, Sri Lanka: International Water Management Institute. 1998. 118p.

Bastiaanssen, W.; Bakker, M. Use of satellite data in agricultural water management. Embrapa Semiárido. Petrolina: 2000. $81 \mathrm{p}$.

Candido, H. G.; Barbosa, M. P.; Silva, M. J. da. Avaliação da degradação ambiental de parte do Seridó Paraibano. Revista Brasileira de Engenharia Agrícola e Ambiental, v.6, n.2, p.368371, 2002.

Carvalho, V. C. Abordagem multiescala para o monitoramento de indicadores do processo de desertificação. In: Simpósio Brasileiro de Sensoriamento Remoto, 10, 2001, Foz do Iguaçu. Anais.... Foz do Iguaçu: SBSR, 2001. p.1539-1551.

Denyer, K.; Burns, B.; Ogden, J. Buffering of native forest edge microclimate by adjoining tree plantations. Austral Ecology, v.31, p.478-489, 2006.

García, M.; Oyonarte, C.; Villagarcía, L.; Contreras S.; Domingo, F.; Puigdefábregas, J. Monitoring land degradation risk using ASTER data: The non-evaporative fraction as an indicator of ecosystem function. Remote Sensing of Environment, v.112, p.3720-3736, 2008.

Gürtler, S.; Epiphanio, J. C. N.; Luiz, A. J. B.; Formaggio, A. R.. Planilha eletrônica para o cálculo da reflectância em imagens TM e ETM+ Landsat. Revista Brasileira de Cartografia, v. 57 n.02, p.162-167, 2005.

Hein, L.; Ridder, N. de. Desertifcation in the Sahel: A reinterpretation. Global Change Biology, v.12, p.751-758, 2006.

Herb, W. R.; Janke, B; Mohseni, O.; Stefan, H. G. Ground surface temperature simulation for different land covers. Journal of Hydrology, v.356, p.327-343, 2008.
Hountondji, Y. C.; Sokpon, N.; Ozer, P. Analysis of the vegetation trends using low resolution remote sensing data in Burkina Faso (1982-1999) for the monitoring of desertification. International Journal of Remote Sensing, v.27, n.5, p.871-884, 2006.

Julien, Y.; Sobrino, J. A. The yearly land cover dynamics (YLCD) method: An analysis of global vegetation from NDVI and LST parameters. Remote Sensing of Environment, v.113, p.329334, 2009.

Julien, Y.; Sobrino, J. A.; Verhoef, W. Changes in land surface temperatures and NDVI values over Europe between 1982 and 1999. Remote Sensing of Environment, v.103, p.43-55, 2006.

Leibovici, D.; Quillevere, G.; Desconnets, J. C. A method to classify ecoclimatic arid and semiarid zones in Circum-Saharan Africa using monthly dynamics of multiple indicators. IEEE Transactions on Geoscience and Remote Sensing, v.45, n.12, p.4000-4007, 2007.

Liu, Y.; Hiyama, T.; Yamaguchi, Y. Scaling of land surface temperature using satellite data: A case examination on ASTER and MODIS products over a heterogeneous terrain area. Remote Sensing of Environment, v.105, p.115-128, 2006.

Lopes, H. L.; Candeias, A. L. B.; Accioly, L. J. de O.; Sobral, M. do C. M. Modelagem de parâmetros biofísicos para desenvolvimento de algoritmo para avaliação e espacialização de risco a desertificação. Boletim de Ciências Geodésicas, v.15, n.4, p.652-668, 2009.

Pereira, R. M. de A.; Araújo Filho, J. A. de; Lima, R. V.; Paulino, F. D. G.; Lima, A. O. N.; Araújo, A. B. de. Estudos fenológicos de algumas espécies lenhosas e herbáceas da caatinga. Ciência Agronômica, v.20, p.11-20, 1989.

Qi, J.; Chehbouni A.; Huete, A. R.; Kerr, Y. H.; Sorooshian, S. A. Modified soil adjusted vegetation index. Remote Sensing Environment, v.48, p.119-126, 1994.

Rahman, S.; Rahman, H.; Keramat, M. Study on the seasonal changes of land cover and their impact on surface albedo in the northwestern part of Bangladesh using remote sensing. International Journal of Remote Sensing. v.28, n.5, p.1001-1022, 2007.

Rubio, J. L.; Bochet, E. Desertification indicators as diagnosis criteria for desertification risk assessment in Europe. Journal of Arid Environments, v.39, p.113-120, 1998.

Santos, J. de S. Uso do sensoriamento remoto no estudo das mudanças da cobertura de vegetação nativa do Município de Araripina-PE. Recife: ITEP, 2006. 71p. Dissertação Mestrado

Silva, S. S.; Solange, E.; Pareyn, F. . Consumo de energéticos florestais do setor industrial/comercial no Estado de Pernambuco. Projeto PNUD/FAO/BRA/87/007/Governo de Pernambuco. Documento de Campo n 15. Recife,PE, 1998.

Souza, B. I. de; Silans, A. M. B. P. de; Santos, J. B. dos. Contribuição ao estudo da desertificação na Bacia do Taperoá. Revista Brasileira de Engenharia Agrícola e Ambiental, v.8, n.2/3, p.292-298, 2004.

UN - General Assembly Report on the United Nations/European Space Agency/Committee on Space Research Workshop on Data Analysis and Image-Processing Techniques Distr.: General 14 August 2001 Original: English. Damascus, 25-29 March 2001 . http://www.oosa.unvienna.org/Reports/ AC105_765E.pdf. 12 Dez. 2003. 
UN - United Nations Convention to combat desertification. Conference of the Parties, Second session. Dakar, 17 November 1998. http://www.geic.or.jp/interlinkages/ docs/ccd.PDF. 03 Out. 2003.
Yan, C. Z.; Wang, T.; Han, Z. W.; Qie, Y. F. Surveying sandy deserts and desertified lands in north-western China by remote sensing. International Journal of Remote Sensing, v.28, n.16, p.3603-3618, 2007. 